\title{
Right N170 modulation in a face discrimination task: An account for categorical perception of familiar faces
}

\author{
S. CAMPANELLA, ${ }^{\mathrm{a}}$ C. HANOTEAU, ${ }^{\mathrm{a}}$ D. DÉPY,${ }^{\mathrm{a}, \mathrm{b}}$ B. ROSSION,,${ }^{\mathrm{a}, \mathrm{c}}$ R. BRUYER, ${ }^{\mathrm{a}}$ \\ M. CROMMELINCK, ${ }^{c}$ AND J. M. GUÉRIT ${ }^{c}$ \\ a Unité de Neuropsychologie Cognitive (NECO), Université Catholique de Louvain, Louvain-la-Neuve, Belgium \\ ${ }^{\mathrm{b}}$ Centre de Recherche en Neurosciences Cognitives, CNRS, Marseille, France \\ ${ }^{c}$ Laboratoire de Neurophysiologie, Université Catholique de Louvain, Brussels, Belgium
}

\begin{abstract}
Behavioral studies have shown that two different morphed faces belonging to the same identity are harder to discriminate than two faces stemming from two different identities. The temporal course of this categorical perception effect has been explored through event-related potentials. Three kinds of pairs were presented in a matching task: (1) two different morphed faces representing the same identity (within), (2) two other faces representing two different identities (between), and (3) two identical morphed faces (same). Following the second face onset in the pair, the amplitude of the right occipitotemporal negativity (N170) was reduced for within and same pairs as compared with between pairs, suggesting an identity priming effect. We also observed a modulation of the P3b wave, as the amplitude of the responses for within pairs was higher than for between and same pairs, suggesting a higher complexity of the task for within pairs. These results indicate that categorical perception of human faces has a perceptual origin in the right occipitotemporal hemisphere.
\end{abstract}

Descriptors: Event-related potentials, Face perceptual categorization, N170, Priming, P300, Task complexity

In 1995, Beale and Keil showed categorical perception of familiar facial identities. By using a morphing technique, they created continua of morphed faces that changed linearly from one identity (U.S. President John F. Kennedy) to another one (U.S. President Bill Clinton). These researchers used a two-step procedure.

First, subjects were confronted with an identification task, during which they had to categorize all the randomly presented morphed faces as either Kennedy or Clinton. Although the identity information contained in the morphed faces was linearly manipulated, there were sharp boundaries in the subjects' responses. Indeed, only morphed faces Clinton $40 \% /$ Kennedy $60 \%$, Clinton $50 \% /$ Kennedy $50 \%$, and Clinton $60 \%$ /Kennedy $40 \%$ induced ambiguous responses whereas other faces, such as Clinton $90 \% /$ Kennedy $10 \%$, Clinton $80 \%$ /Kennedy $20 \%$, or Clinton $70 \% /$ Kennedy $30 \%$, were clearly referred to the identity predominantly represented in the morphed face (in this case, Clinton). Tanaka, Giles, Kremen, and Simon (1998) recently proposed a theoretical interpretation of this observation. According to Tanaka et al., morphed faces situated close to the midpoint of the continua (50\%) defined the categorical boundary, that is, the point at which $50 \%$ of

This study was supported by grant 95/00-189. S. Campanella, D. Dépy, and B. Rossion are supported by the Belgian Fund of Scientific Research (F.N.R.S.)

Address reprint requests to: Salvatore Campanella, Faculté de Psychologie-Unité NECO, Université Catholique de Louvain, Place du Cardinal Mercier, 10, B-1348 Louvain-la-Neuve, Belgium. E-mail: campanella@ neco.ucl.ac.be. the subjects answered "Clinton" whereas the other half answered "Kennedy". On the other hand, morphed faces situated close to the extremities of the continua were attracted by their respective prototype representations, which are stored in long-term memory.

In the second step of their procedure, Beale and Keil (1995) confronted subjects with an $A B X$ discrimination task, in which two morphed faces (A, B) were successively presented, followed by a third one $(\mathrm{X})$ that they had to match to either A or B. Given that the identification task defined the categorical boundary of the continuum, A-B within-categorical (within) and A-B betweencategorical (between) pairs were designed in such a way that two morphed faces attracted by the same prototype representation composed the within pairs and two morphed faces attracted by two different ones composed the between pairs. Although the physical distance between the stimuli of each pair was kept constant (20\%), subjects better discriminated between pairs than within pairs. In other words, subjects discriminated more easily two morphed faces belonging to two different identities than two morphed faces belonging to the same one. This facilitation effect on face discrimination is better known as the categorical perception effect on familiar faces (Harnad, 1987). Categorical perception needs, then, two steps to be assessed: (1) an identification task, which has to show nonlinear responses to linearly manipulated stimuli and which allows definition of boundaries within each continuum, and (2) a discrimination task, which defines the hallmark of categorical perception effect (Young et al., 1997) and which has to evidence an enhanced discriminability for between - as compared with withincategorical differences. 


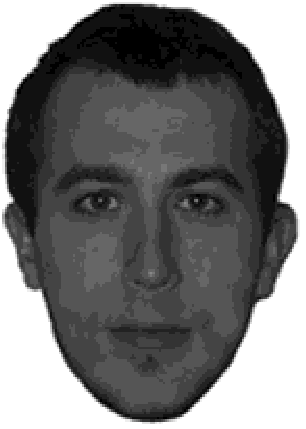

A

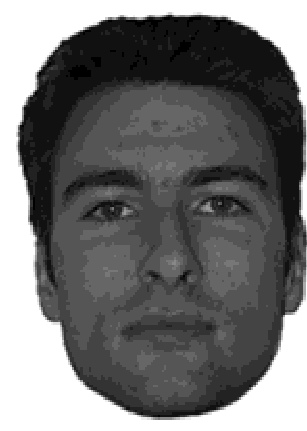

B

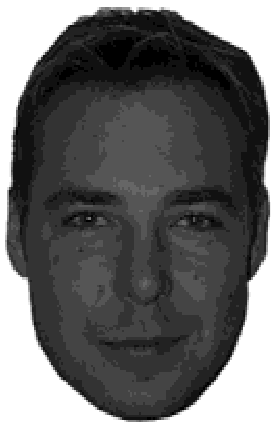

C

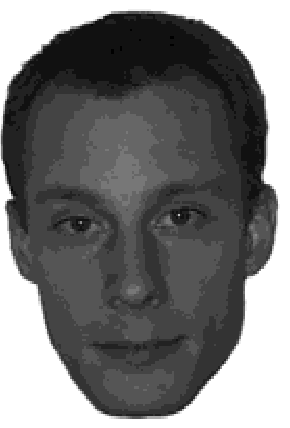

D

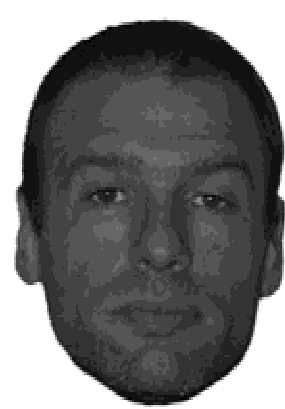

$\mathbf{E}$

Figure 1. Photographed source faces of five men

Categorical perception effect was initially observed on unidimensional stimuli, such as speech sounds and color perception (Bornstein \& Korda, 1984; Liberman, Harris, Hoffman, \& Griffith, 1957). Even if humans are confronted with physical linear changes, they perceive both phonemes and hues categorically. This highly specific psychophysical phenomenon is of the greatest relevance as it may provide a representative model for the categorization process in general (Harnad, 1987). Indeed, even though the ability to recognize specific individuals must be learned and the continua between individual faces are not naturally occurring, there might be general constraints on category formation that apply also to individual face recognition. Recent developments and applications of computer image-manipulation techniques made the investigations of multidimensional stimuli, such as the human face, possible. Thus, during the last few years, several behavioral studies provided empirical support for categorical perception of familiar faces (Beale \& Keil, 1995; Stevenage, 1998). Tanaka et al. (1998) proposed that the categorical perception effect observed in faces is due to the way faces are stored in memory, relative to a facial prototype (Valentine, 1991). Accordingly, categorical perception is correlated with face familiarity (Beale \& Keil, 1995) and should not be observed with unfamiliar faces (Goldstone, 1998).

The aim of the present study is to give a neurophysiological account of the categorical perception effect on familiar faces by using event-related potentials (ERPs). More precisely, we used ERPs to investigate where and mainly when does the categorical perception of faces occur. Indeed, ERPs allow us to investigate the temporal course of face processing from stimulation to motor response. Moreover, scalp recordings of ERPs specific to faces have distinguished component processes specific to face perception over the last decade (Bentin, Allison, Puce, Perez, \& McCarthy, 1996; Jeffreys, 1996). It seems thus particularly well suited to clarify the temporal and functional origin of the categorical perception effect. Using this method, Dehaene-Lambertz and Dehaene (1994) demonstrated in 2-month old infants that categorical perception of speech began at $220 \mathrm{~ms}$ following the stimulus onset. A similar observation in the present study would involve a modulation of face-specific neural processing, which is supposed to take place around $170 \mathrm{~ms}$ in occipitotemporal regions (Bentin et al., 1996) and is not influenced by face familiarity at this stage (Bentin \& Deouell, 2000; Rossion, Campanella, et al., 1999).

Pairs of faces were then presented to the subjects. Those pairs were composed of morphed faces that were exactly the same or differed from a defined step $(30 \%)$ in a continuum but were either categorized as the same identity or as two different identities.

\section{Materials and Methods}

\section{Stimuli}

Five male faces (M1-M5) were photographed (Figure 1). Ten continua of pairs were therefore possible as each male face paired with the four others.

Five morphed images were created for each continuum. They were prepared by blending two faces in the following proportions: 90:10 (i.e., 90\% M1 and 10\% M2), 70:30, 50:50, 30:70, and 10:90. We will refer to these as $90 \%, 70 \%, 50 \%, 30 \%$, and $10 \%$ morphs along the appropriate continuum (Figure 2).

The preparation of each continuum involved five steps. First, photographic quality images (digital camera) of faces were chosen as source images. The subjects did not wear beards, moustaches, or glasses. All faces were fully frontal and with a neutral facial expression. Second, these photographs were downloaded into a Macintosh computer (Apple Computer, Inc.) and were edited by Adobe Photoshop 4.0.1(r) (Adobe Systems, Inc.) to remove backgrounds and everything below the chin. Gray-scale images were created and scaled to $150 \times 191$ pixels. Third, morphed stimuli were generated using the Morph 2.5(r) program (Gryphon Software Corp.). One hundred fifty points were located manually onto the sources. The locations of these points were specified in terms of facial features such as corners of the mouth, tip and bridge of the nose, or outlines of the eyes. The same method was applied to the other sources so that there was a correspondence of the 150 points for all faces. Fourth, a vector equation for each of the 150 points was computed on the sources to determine which position a point on M1's face will have on the morphed image after moving to $10 \%, 30 \%, 50 \%, 70 \%$, or $90 \%$ to the position of the corresponding point on M2's face. Fifth, the Morph program used a warping procedure to move from one source to the other by allowing the shift of the 150 control points from their initial position (in one source) to their final position (in the other) along linear changes. For example, in the $90 \% \mathrm{M} 1 / 10 \% \mathrm{M} 2$ morphed face, the pixel intensities have deformed the M1's face by $10 \%$ toward the M2's face and the M2's face by $90 \%$ toward the M1's face. In total, 50 images were drawn ( 5 from each of the 10 continua). ${ }^{1}$ These faces were converted to a PCX format to be displayed on a monitor using a commercial visual stimulator (STIM, Neuroscan SCAN(r), Neuromed Holland, Inc.).

${ }^{1}$ More precise details on the morphing technique are available in Young et al. (1997). 


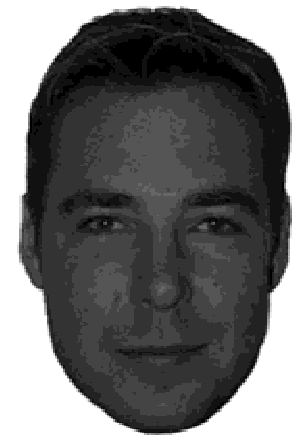

BC $10 \% / 90 \%$

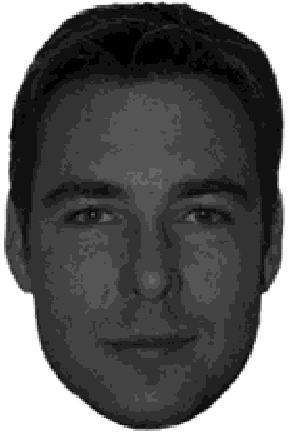

BC $30 \% / 70 \%$

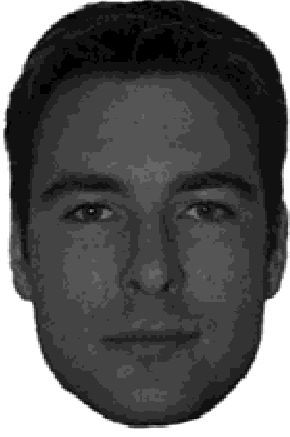

BC $\mathbf{5 0} \% / \mathbf{5 0} \%$

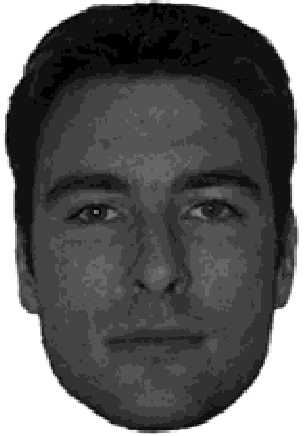

BC $30 \% / 70 \%$

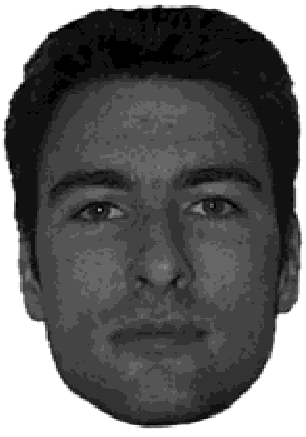

BC $10 \% / 90 \%$

Figure 2. Illustration of the morphed faces for the continuum B-C.

\section{Behavioral Preexperiments}

The aim of this behavioral experiment was to identify the categorical boundary of the 10 face continua in order to prepare pairs of faces for the ERP experiment. Twelve subjects were familiarized with the five previously unknown source faces. The familiarization phase consisted of three stages:

1. for each of the five faces, subjects were confronted with a full-frontal, a right and left $3 / 4$ profiles, a right and left profile presented alone, and then with an image grouping these five representations (Figure 3) on a black-and-white screen monitor. Subjects observed all these images three times and $15 \mathrm{~s}$ each (for a total of $45 \mathrm{~s}$ ). After the onset of the presentation, the experimentor gave the name, the profession, and the age of the presented face to the subjects. They were asked to encode the face in memory, linked with these pieces of semantic information.

2. In a second stage, all the encoded faces were presented randomly one at a time to subjects who had to recall the information previously learned. The experimentor helped them if they failed: this step came to an end when subjects were able to associate the correct information to each presented image.

3. In a third stage, subjects were confronted with a familiarity task in which they had to decide whether the observed face was present in the previously learned set or not (this set was constituted by all the images described in Step 1 [above] and by the same number of distractors). Subjects had to reach a performance of $100 \%$ at the familiarity task to go further in the experiment.

Finally, categorical boundaries of the 10 continua were determined by using an identification task. Subjects were confronted with the 50 morphed faces, presented in a random order. Their task consisted of deciding to which identity (M1 to M5) the presented morphed face was more similar. As shown previously (Beale \& Keil, 1995), this task allows us to define for each of the 10 continua the categorical boundary, which is necessary to create between and within pairs (i.e., pairs that respectively passed through or not the categorical boundary) that will be used in the delayed same-different matching task during the ERP recording. The generation of these pairs is necessary to be able to assess in a discrimination task an enhanced discriminability for between-categorical differences as compared with within differences.

\section{Procedure}

Figure 4 shows how the results of the behavioral pretests were processed to identify the categorical boundary of the 10 continua. Each continua gave rise to four pairs of morphed faces: a between pair (for example, a pair in which the first image was a morph identified as M1 and the second one as M2), a within pair (two different morphed images representing both M2, for instance), and two same pairs for methodological purpose (the same image pre-

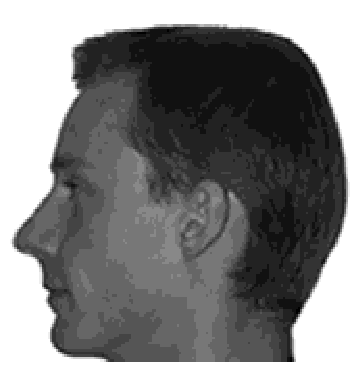

left profile

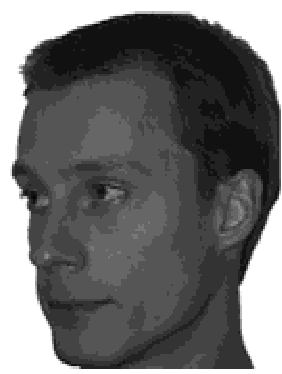

left $3 / 4$ profile

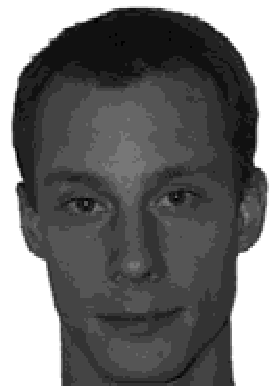

front

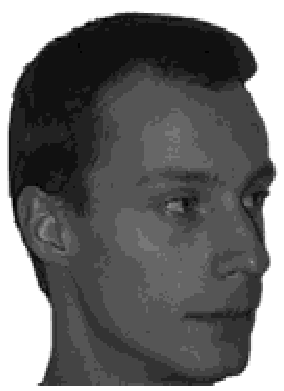

right $3 / 4$ profile

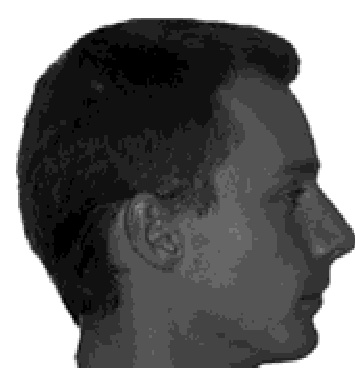

right profile

Figure 3. Illustration of the five different views of D used in the familiarization task. 


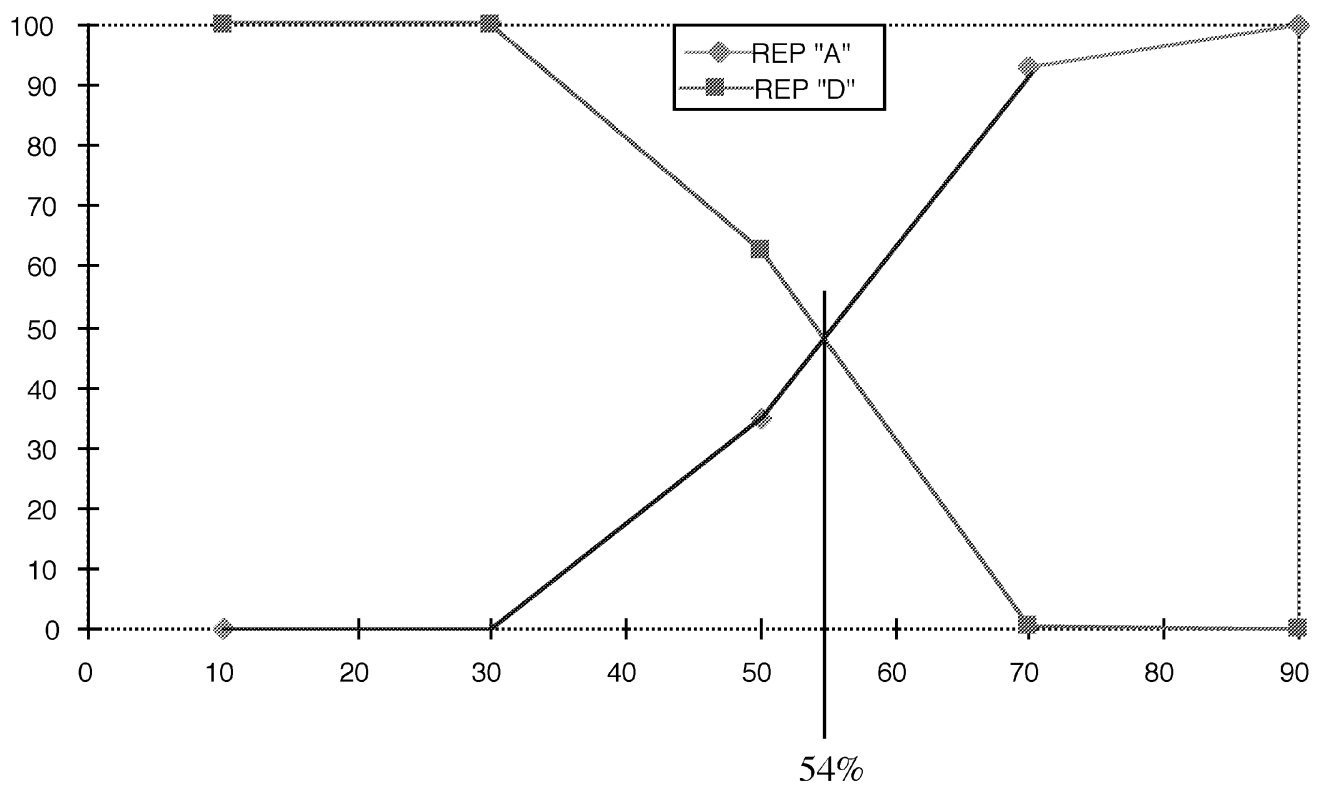

Figure 4. For the continuum A-D, mean frequencies of responses "A" and "D" were calculated. The intersection point of the two curves gives a point corresponding to the morphed face $54 \%$. This point indicates the categorical boundary of this continuum, that is, the subjective point where $50 \%$ of the subjects responded "A" and the other half responded "D." The same procedure was applied to the other nine continua. Note the similarity between the results of the "pilot study" group and the experimental one.

sented twice). Indeed, we made same pairs in order to have an equivalent number of same and different pairs to present to subjects. Note that the physical difference between the stimuli of between and within pairs was kept equivalent (30\%) (Figure 5).

A total of 40 pairs was available (20 same pairs and 20 different pairs with 10 between and 10 within pairs). To increase the signalto-noise ratio, these pairs were repeated eight times, so that 320 trials (160 same, 80 between, and 80 within) were recorded. All stimuli were used equally often in each of the three conditions.

During the electroencephalographic (EEG) recording, subjects sat on a chair in a dark room with their head restrained by a chin rest. Their heads were placed $1 \mathrm{~m}$ from the screen, and stimuli were $6 \mathrm{~cm}$ horizontal and $8 \mathrm{~cm}$ vertical; stimuli thus subtended a visual angle of $10.5^{\circ} \times 14.3^{\circ}$. Subjects were presented with 16 blocks of 20 pairs of stimuli, the between, within, and same pairs being randomly intermixed within each block of trials. The order of the 16 blocks was also counterbalanced across subjects. The beginning of each trial was signaled by a small white cross that remained on the center of the screen for $300 \mathrm{~ms}$ followed then by a black screen for $400 \mathrm{~ms}$. Then, the first image was presented for $400 \mathrm{~ms}$. A black screen was displayed for 1,300 ms before the onset of the second image for $400 \mathrm{~ms}$. The intertrial interval was set at 1,500 ms (black screen), but subjects had 1,200 ms after the second stimulus onset to answer. The participants had to decide as quickly and as accurately as possible whether the second image of the presented pair was exactly the same as the first one (delayed same-different matching task). This task shares the same goal as the $\mathrm{ABX}$ discrimination task used in the categorical perception literature, that is, to show an enhanced discriminability for beweencategorical differences as compared with within-categorical differences, with the advantage that memory load component is reduced. Subjects had to press the right or left key on a mouse with the right finger. The labeling (same/different) of the buttons was counterbalanced across subjects.

\section{Subjects}

Twelve new participants (right-handed men, 21-26 years, without neurological disease and with normal/corrected vision) volunteered for cash in the ERP experiment. They were also familiarized with the five previously unknown faces (see the three stages described above) before starting the ERP recording.

\section{EEG Recordings}

EEG was recorded by 58 electrodes mounted in an electrode cap. Electrode positions included the standard International 10-20 system locations and additional intermediate positions. Recordings were made with a left ear physical reference. The EEG was amplified by battery-operated SYNAMPS (r) (Neuromed Holland, Inc.) amplifiers with a gain of 30,000 and a bandpass of 0.01-100 Hz. The impedance of all electrodes was kept below $5 \mathrm{k} \Omega$. EEG was continuously recorded (sampling rate $500 \mathrm{~Hz}$, Neuroscan) and stored on disk for further analyses. Electrooculographic artifacts were eliminated and epochs beginning $100 \mathrm{~ms}$ prior to stimulus onset and continuing for $924 \mathrm{~ms}$ were created. A recalculation was made to obtain common average reference recordings (Bertrand, Perrin, $\&$ Perrier, 1985). Codes synchronized with stimulus delivery were used to average selectively epochs associated with different stimulus types. Three parameters were coded for every stimulus: (1) the position in the pair (first or second image); (2) the type of the pair (between, within, same); and (3) the response type (same, different). This coding allowed us to compute different averages of ERP target stimuli. These averages were made for each subject individually. A sample grand average was obtained by averaging across the subjects the averages for each experimental conditions, that is, the first and the second face of a between pair (BET1 and BET2), of a within pair (WIT1 and WIT2), and of a same pair (SAM1 and SAM2). Only correct trials were included in averages of BET2, WIT2, and SAM2. Finally, the data were filtered from 1 to $30 \mathrm{~Hz}$. 

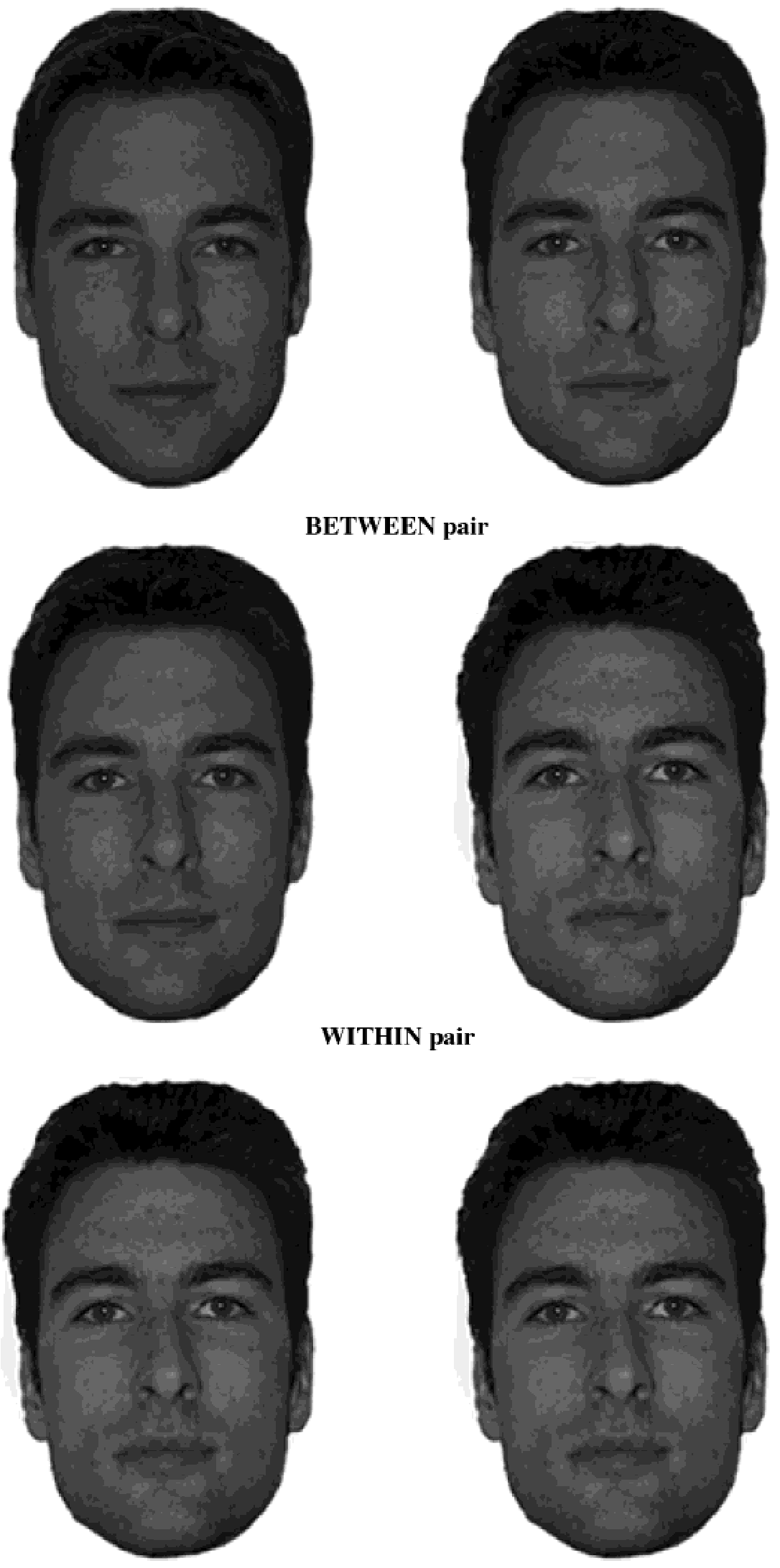

SAME pair

Figure 5. Pairs of morphed faces which crossed (between) or not (within) the boundary were generated for each continuum. Pairs of identical stimuli (same) were also created for methodological purposes. 


\section{Statistical Analyses}

Correct latencies and percentages of correct responses were computed and analyzed with Systat 5.1(r) (Systat, Inc.). Three subjects did not reach the threshold of $70 \%$ of correct responses for the same pairs and one did not reach this threshold for the different pairs (mean of between and within pairs). As a consequence, their data were not taken into account. All further analyses were then computed on eight subjects. At selected electrodes, peak amplitudes (mean over a 20-ms time window around the peak) of different components were obtained for the different conditions and for each subject individually. These values were tested using paired $t$ tests and repeated-measures of analyses of variance (ANOVAs).

\section{Results}

\section{Behavioral Data}

Our aim was to replicate the categorical perception effect described in the literature, that is, a better performance for between pairs than for within pairs. This performance was analyzed statistically by a three-way ANOVA with condition (between, within, and same) as the dependent variable, on the percentages of correct responses. The results revealed a clear effect of the condition, $F(2,14)=17,976, p<.0001$. Moreover, further paired Student $t$ tests showed a better performance for between as compared with within pairs, $t(7)=5,144, p=.001$, whereas subjects showed a better performance for same pairs as compared with within pairs, $t(7)=4,192, p=.004$, and no difference between same and between pairs, $t(7)=0,538, n s$. Note that this pattern of results was not found by considering correct responses latencies, ANOVA 3: $F(2,14)=2,408, n s$. These results suggest that subjects discriminated more easily two faces belonging to two different identities (between pairs) than two faces belonging to the same one (within pairs), even if the physical difference inside each pair was kept constant. Moreover, a same pattern of performance was observed for between and same pairs (Table 1).

\section{Event-Related Potentials}

In response to the second face of each pair (BET2, WIT2, and SAM2), three clear components were observed for all subjects in all conditions (except for a few exceptions, see below) (Figure 6B). These electrophysiological events were named according to their order of occurrence and polarity as an occipitocentral bipolar P1/N1 complex, the N170 (Bentin et al., 1996) synchronized with the vertex positive potential (VPP, Jeffreys, 1996), and a posterior long-lasting positivity, which was identified as a P300. Note that the P300 was not observed in response to the first face of each pair, whereas P1/N1 and N170/VPP were both observable (Figure 6A).

\section{The P1/N1 Complex}

The first measurable electrophysiological event was a bipolar complex $\mathrm{P} 1 / \mathrm{N} 1$, which showed a large positivity over all posterior

Table 1. Mean Correct Responses and Mean Correct Latencies for Same, Within, and Between Pairs

\begin{tabular}{lrrr}
\hline \hline & Same & Between & Within \\
\hline Mean correct responses (\%) & 89 & 89 & 73 \\
Mean correct latencies (ms) & 698 & 708 & 744 \\
\hline \hline
\end{tabular}

electrodes culminating $(\mathrm{Oz})$ at $112 \mathrm{~ms}$ and with polarity reversal at central sites $(\mathrm{Cz})$ (Figure 7A). The P1 was present in seven of the eight subjects whereas the N1 counterpart was present in all eight subjects.

There were no significant differences between the three conditions (between, within, and same) for voltage amplitude of P1 at $\mathrm{Oz}$, neither for the responses to the first face of the pairs, BET1 vs. WIT1: $t(6)=0,194, n s$; BET1 vs. SAM1: $t(6)=0,513, n s$; WIT1 vs. SAM1: $t(6)=0,498, n s$, nor to the second face of the pairs, BET2 vs. WIT2: $t(6)=1,247, n s$; BET2 vs. SAM2: $t(6)=0,615$, $n s$; WIT2 vs. SAM2: $t(6)=0,298, n s$.

An ANOVA on peak amplitudes with condition (between, within, same) and lateralization (O1-O2) as factors did not reveal any significant effect, neither for the responses to the first face of the pairs, condition: $F(2,12)=0,786, n s$; lateralization: $F(1,6)=$ 0,287, $n s$; interaction: $F(2,12)=0,048, n s$, nor to the second face of the pairs, condition: $F(2,12)=0,324, n s$; lateralization: $F(1,6)=$ 1,195, $n s$; interaction: $F(2,12)=3,085, n s$.

An identical analysis was performed on the negative counterpart of the $\mathrm{P} 1$ at $\mathrm{Cz}$ at the same latency. The same pattern of results was found for the responses to the first image of the pairs, condition: $F(2,14)=0,505, n s$; lateralization $(\mathrm{C} 1-\mathrm{C} 2): F(1,7)=$ 2,252, $n s$; interaction: $F(2,14)=0,919, n s$, as well as for the second image of the pairs, condition: $F(2,14)=2,210$, $n s$; lateralization: $F(1,7)=1,112$, ns; interaction: $F(2,14)=$ $2,377, \mathrm{~ns}$

\section{The N170 and the VPP}

The next major electrophysiological event corresponded to the N170, which was observable bilaterally in all subjects, culminated at T5/T6 electrodes at $156 \mathrm{~ms}$, and was synchronized with a vertex positive potential maximally recorded at $\mathrm{Cz}$ at $158 \mathrm{~ms}$ (Figure 7B). The N170 appeared to be similar in latency and amplitude for the different conditions when the first face of the pairs was taken into account. However, the amplitude of the N170 was reduced when the second face was identical (same) or belonged to the same identity as the first face (within) as compared to the amplitude of the N170 following a face that belonged to a different category than the first face (between). This amplitude reduction was observed only in the right hemisphere.

Statistical analyses confirmed these observations. First, an ANOVA with condition (between, within, same) and lateralization (left T5, right T6) as factors failed to find any significant difference for the responses to the first face of the pairs, condition: $F(2,14)=0,181, n s$; lateralization: $F(1,7)=1,49, n s$; interaction: $F(2,14)=0,773, n s$. However, the same ANOVA found a clear main effect of condition, $F(2,14)=4,186, p=.038$, a significant interaction, $F(2,14)=5,542, p=.017$, and no main effect of lateralization, $F(1,7)=0,414$, $n s$, when responses to the second images of the pairs were considered. Post hoc Student $t$ tests showed a significant difference between within pairs and between pairs at T6, $t(7)=3,799, p=.007$, and between same pairs and between pairs at T6, $t(7)=3,118, p=.017$, whereas these differences were not observable at T5, between vs. within: $t(7)=0,086, n s$; between vs. same: $t(7)=2,322, n s$. Moreover, no difference was found between same and within pairs at T5, $t(7)=1,623, n s$, and at T6, $t(7)=2,205, n s$ (Figure 8).

To sum up, in contrast to behavioral results, in which an equally good performance for between and same pairs was found (while within pairs were harder to discriminate), ERPs to the second face of the pairs showed that the N170 was reduced for within and same pairs as compared with between pairs. 

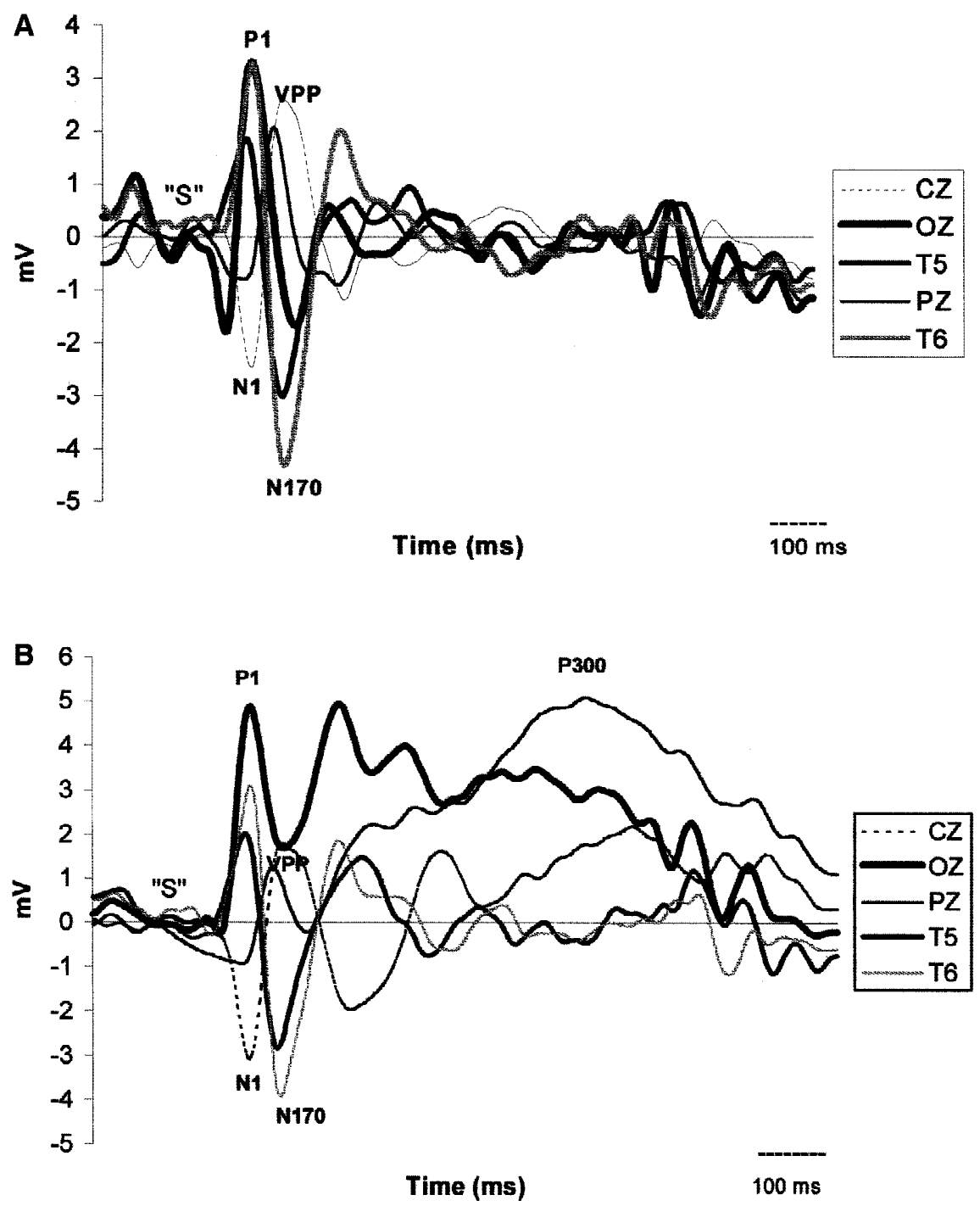

Figure 6. Illustration of the event-related brain potentials recorded from T6, T5, OZ, CZ, and PZ in response to the first (A) and the second face (B) of between pairs. Note the appearance of the P300 only in response to the second face of the pairs.

Similar analyses were performed on the VPP observable for seven of the eight subjects. An ANOVA with condition (between, within, and same) and lateralization $(\mathrm{C} 1, \mathrm{C} 2)$ was applied for response amplitudes to the second image of the pairs and gave rise to a different pattern of results from the one found for the N170. Indeed, a main effect of lateralization, $F(1,6)=8,64, p=.026$, emerged for the right hemisphere, whereas condition factor and interaction did not show any significant differences, condition: $F(2,12)=3,187, n s$; interaction: $F(2,12)=3,631, n s$.

\section{The P300}

The N170/VPP complex was followed by a P300, which was maximally recorded at $\mathrm{Pz}$ and which began around $250 \mathrm{~ms}$. This P300 was observed for all subjects in response to the presentation of the second face of the pairs and was absent in response to the first face of the pairs, which did not require any subject response (Figure 7C). This finding is in agreement with the functional role assigned to the P300 component, which is regarded as reflecting a decision-making process (Halgren et al., 1994; Rohrbaugh, Donchin, \& Eriksen, 1974).
On the average waveforms, the P300 appeared to be larger in the within condition than in the between and same conditions. The mean amplitude of this P300 was obtained for each subject by averaging the amplitude points in the window 250-550 ms. An ANOVA was performed on these mean values with condition (between, within, same) and lateralization (P1, P2) as factors. Results showed a main effect of condition, $F(2,14)=5,944, p=.014$, whereas lateralization and interaction were not significant, lateralization: $F(1,7)=2,988, n s$; interaction: $F(2,14)=1,249, n s$. Post hoc Student paired $t$ tests showed that within pairs had significant P300 differences as compared with between pairs, $t(7)=$ $3,582, p=.009$, and same pairs, $t(7)=3,044, p=.019$, whereas no difference existed between same and between pairs, $t(7)=$ $0,738, n s$.

\section{Summary of Results}

Overall, it is interesting to note that (1) no significant differences were observed for the P1/N1 complex and (2) contrary to the P300, which showed a pattern of results ( same $=$ between $\neq$ within) correlated with the behavioral results, N170 and VPP brain 


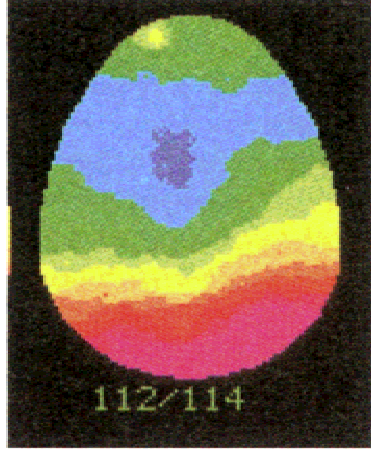

A

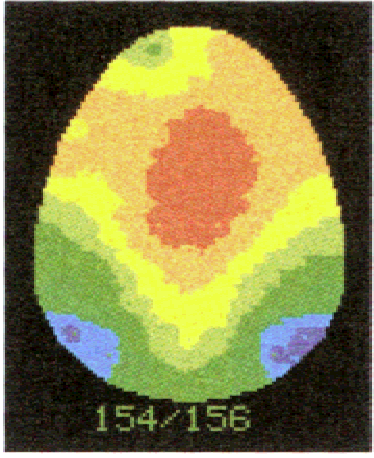

B
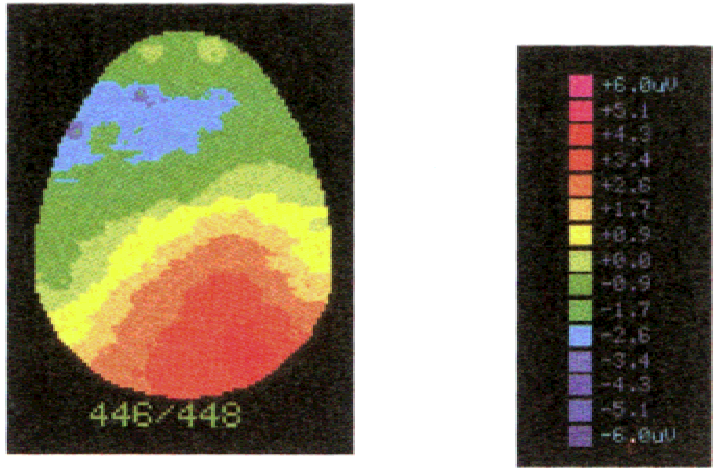

C

Figure 7. Grand-average brain topography of the complex P1/N1 (A), the N170/VPP (B), and the P300 (C) in response to the second face of within pairs

responses to the second face of the pairs showed patterns of responses that were different from those observed in behavioral results (Table 2).

\section{Discussion}

\section{Behavioral Data}

Even if subjects were confronted with pairs of different stimuli separated by a same amount of physical difference $(30 \%$ on the continua), they discriminated more easily between pairs than within pairs. The categorical perception effect described by Beale and Keil in 1995 was thus replicated: this facilitation effect means that subjects discriminated more easily two faces belonging to two different identities than two faces belonging to the same identity, even if the faces were separated by an identical physical distance. Moreover, there was no difference between same and between pairs: this finding suggests that, for subjects, it was as easy to respond to same pairs as to between pairs. The within pairs seemed more complex to respond to and, thus, were regarded as qualitatively different from the same and between pairs of faces.

\section{Event-Related Potentials}

Three complexes - the P1/N1, the N170 synchronized with a VPP, and the P300-were clearly obtained in response to the second

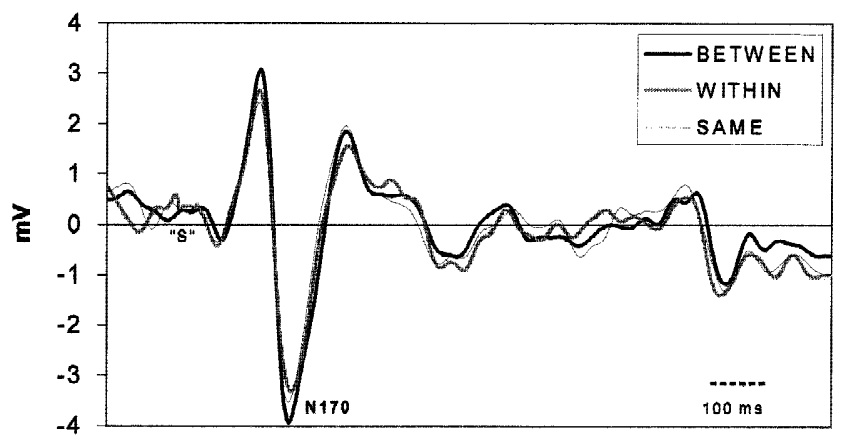

Time (ms)

Figure 8. Illustration of the difference observed on the N170 between within, same, and between pairs at T6. face of the pairs for all the conditions (between, within, same) in almost all subjects. Only one of these complexes, the N170/VPP, is usually considered as face-specific (Bentin et al., 1996; Bentin, Deouell, \& Soroker, 1999; Eimer \& McCarthy, 1999; George, Evans, Fiori, Davidoff, \& Renault, 1996; Jeffreys, 1996; Rossion, Campanella, et al., 1999; Schendan, Ganis, \& Kutas, 1998).

\section{The P1/N1 Complex}

The first positive peak observed at $\mathrm{Oz}$ corresponds to the P1 and the centrofrontal $(\mathrm{Cz})$ negativity occurring with a similar latency $(112 \mathrm{~ms})$ is considered as its dipolar negative counterpart (Bötzel, Schulze, \& Stodieck, 1995). A striate and extrastriate origin is proposed for the $\mathrm{P} 1 / \mathrm{N} 1$ component, which is typically described in visual ERP studies as reflecting primary visual analyses (Gomez, Clarck, Luck, Fan, \& Hillyard, 1994; Heinze et al., 1994). As suggested by statistical analyses, there was no significant difference between conditions (same, within, between pairs) in response to the first image of each presented pair. At first glance, this absence of difference is not surprising as all stimuli were of identical luminosity, contrast, and complexity. Nevertheless, this absence of effect had to be proved to ensure (1) that the signalto-noise ratio, and thus the quality of the ERP recording, was of good quality and (2) that, regardless of the condition, subjects processed in the same way the first images of each pair. Indeed, Clark and Hillyard (1996) showed that early visual responses could be modulated by attentional processes. It was important to observe that, in the present study, repetitions of faces did not produce attentional effects.

Moreover, any significant difference in response to the second face of each pair was found on the P1/N1. This finding suggests

Table 2. Synthesis of the Significant Differences Found by Comparing Pairs for the Behavioral Data and the Evoked Brain Response P1/N1, N170, VPP, and P300

\begin{tabular}{lccccc}
\hline \hline & Behavioral & P1/N1 & N170 & VPP & P300 \\
\hline Same vs. Within & $\neq$ & - & - & - & $\neq$ \\
Within vs. Between & $\neq$ & - & $\neq$ & - & $\neq$ \\
Same vs. Between & - & - & $\neq$ & - & - \\
& & & & & \\
\hline \hline
\end{tabular}


that the early visual processes indexed by the P1/N1 complex are not modulated by the fact that the second face of the pairs was issued (same or within pairs) or not (between pairs) from the same category as the first face of the pairs.

\section{The N170/VPP Complex}

Several studies have described a N170 component to faces with characteristics (latency, amplitude, and topography) comparable to the ones described in this study (Bentin et al., 1996; George et al., 1996; Rossion, Delvenne, et al., 1999). Also in keeping with other studies, the N170 recorded at temporal sites (T5 and T6) reversed polarity at the level of $\mathrm{Cz}$ to give rise to an activity better known as the VPP (see Bötzel \& Grüsser, 1989; Bötzel et al., 1995; Jeffreys, 1989; Jeffreys \& Tukmachi, 1992; Rossion, Campanella, et al., 1999; Rossion, Delvenne, et al., 1999). First, we will discuss the effects observed on the N170. Second, we will focus on the relation between the N170 and the VPP. Indeed, even if these two activities are considered to be face specific, whether the VPP is the positive counterpart of the N170 or not is still a matter of debate (George et al., 1996; Rossion, Campanella, et al., 1999; Rossion, Delvenne, et al., 1999).

The N170 modulation. The results showed that the N170 was identical for all the conditions when the first stimulus of the pair was taken into consideration. The N170 is considered as the process indexing the structural analysis of facial information in order to obtain a configurational face representation (Jeffreys, 1996). The absence of N170 differences in response to the first face of between, within, and same pairs suggests an identical configurational analysis of these faces.

However, considering N170 responses to the second face of these pairs, the results showed (1) that the N170 is larger for between pairs than for within and same pairs; and (2) that this effect is strictly right lateralized. The higher amplitude of the N170 in response to the second face of between pairs as compared with within and same pairs (for which no N170 difference emerged) can be understood if we consider that subjects are confronted with two faces (in between pairs) categorized as two different identities by the perceptual system. Two different configurational facial analyses have thus to be performed successively in the between condition whereas in the within and same conditions, the second face belongs to the same identity as the first one. Several ERP studies have shown that successive repetitions of words, objects, and faces lead to a reduction in ERP amplitudes (Paller \& Gross, 1998; Schweinberger, 1996; Zhang, Begleiter, Porjesz, \& Litke, 1997). In particular, regarding face processing, repetition priming effects on ERPs-indexed by a lower amplitude to the second face presentation-have been observed (Begleiter, Porjesz, \& Wang, 1995; Ji, Porjesz, \& Begleiter, 1998). These repetition priming effects can modulate both early (around $150 \mathrm{~ms}$ ) and late ERPs (beyond $300 \mathrm{~ms}$ ) (Schweinberger, Pfutze, \& Sommer, 1995) and are often right lateralized (Badgaiyan \& Posner, 1996).

Considering this evidence, we propose that the striking reduction in the N170 amplitude to the second face of the same and within pairs reflects an identity-priming effect. Indeed, between pairs comprise two faces belonging to two different identities, whereas same and within pairs refer to two same identities. According to this suggestion, the priming effect indicates that the perceptual system considered the two physical different faces of the within pairs as belonging to an identical facial identity. In other words, faces judged to be identical produce identity priming whereas faces judged to be not identical produce no (or less) priming. Then, these data could suggest that the priming effect affecting the N170 had a conceptual origin and define a conceptual priming on early (extrastriate) visual mechanisms.

Besides, we also showed that this priming effect is right lateralized. This observation is in agreement with the right hemisphere advantage in face processing, as demonstrated by neuropsychological observations (e.g., Landis, Cummings, Christen, Bogen, \& Imhof, 1986; Rapcsak, Polster, Glisky, \& Comer, 1996), divided visual field stimulations (e.g., Hillger \& Koenig, 1991), intracranial ERP recordings (Allison, Puce, Spencer, \& McCarthy, 1999), and neuroimaging studies (e.g., De Renzi, Perani, Carlesimo, Silveri, \& Fazio, 1994; Kanwisher, McDermott, \& Chun, 1997; McCarthy, Puce, Gore, \& Allison, 1997). Moreover, the face N170 is maximally recorded at the right hemisphere (Bentin et al., 1996; Bötzel et al., 1995; George et al., 1996) and has been found to be more sensitive to face transformations in the right hemisphere (e.g., Jemel, George, Chaby, Fiori, \& Renault, 1999; Rossion, Delvenne, et al., 1999). Finally, auditory-evoked potentials (Dorman, 1974) and clinical neuropsychology literature (Blumstein, Cooper, Zurif, \& Caramazza, 1977) suggest a particular role played by the right hemisphere in the process of categorical perception (see Molfese, 1987, for review).

The relation between $N 170$ and VPP. In the present study, we also observed different patterns of results for the N170 and the VPP. Indeed, a clear main effect of condition and a significant interaction of Condition $\times$ Lateralization were described for the N170 whereas a clear main effect of lateralization emerged for the VPP. In other words, whereas N170 analyses disclosed a right-lateralized clear effect of the type of pair (between, within, same), VPP analyses only suggest a greater amplitude of the VPP to the right hemisphere. This dissociation may be surprising at first glance if we consider the central positive potential (VPP) as reflecting (at least mainly) the counterpart of the bilateral negative activities (N170) observed at temporal sites (George et al., 1996; Rossion, Campanella, et al., 1999; Rossion, Delvenne, et al., 1999). Accordingly, an experimental effect observed on the temporal negativities would be reflected as a similar effect on the VPP, as shown for example by the N170 modulation for face inversion, which is observed at both components (Rossion, Delvenne, et al., 1999).

However, it seems highly plausible that the closer the current sources are from the electrodes, the larger the differences between two experimental conditions will be observed. To strenghten this suggestion, it is useful to note that the VPP is always larger for faces than objects (Jeffreys, 1996), but the amplitude difference does not seem to be as large as for the N170, an observation that led some researchers to suggest that the N170 is even not observed for objects (Bentin et al., 1996; Bentin, Deouell, \& Soroker, 1999). Moreover, the topography of the N170 suggests a potential distribution produced by tangentially oriented dipole generators directly beneath the site of the polarity reversal, that is, from posterior temporal lobes (Jeffreys, 1989; Taylor, McCarthy, Saliba, \& Degiovanni, 1999). Thus, because the VPP is supposed to be far from the sources (contrary to the N170 recorded at T5-T6) and because the effects we observed on the N170 were limited to the right hemisphere, it seems that some effects generated by right posterior temporal regions could be detected only by closer electrodes (in this case, T6). Nevertheless, these results may not be considered as an argument against the view that these two potentials reflect an identical brain activation. 


\section{The P300}

The long-lasting positivity described in response to the second face of between, within, and same pairs has the same latency, amplitude, and topography as the P3b activity described in other studies and referring to a decision-making process (Bentin, Mouchetant-Rostaing, Giard, Echallier, 1999; Halgren et al., 1994; Rohrbaugh et al., 1974). Indeed, several studies have emphasized the distinction between a frontocentral ("P3a") and a parietal ("P3b") component of the P300. In the present study, the centroparietal distribution of the P300 identified it as a P3b (see Figure 7C). This identification is in agreement with the fact that no P3b was observed in response to the first face of each pair, any response (decision) being expected after this image presentation.

As suggested by statistical analyses, the P3b showed a greater activity for within pairs in comparison with to same and between pairs. It is of particular interest that these P3b modulations were correlated with behavioral results. This effect can be interpreted in conjunction with results shown on N170 and by considering task demands. Indeed, it is well known that P3b amplitude is modulated by task complexity (Comerchero \& Polich, 1999; Duncan-Johnson \& Donchin, 1982; Ravden \& Polich, 1998; Wilson, Swain, \& Ullsperger, 1998). For instance, a recent study (Comerchero \& Polich, 1999) showed, by using an oddball paradigm, that when target/standard discrimination was difficult, the target amplitude (P3b) was larger parietally and occurred later than nontarget components, for both visual and auditory stimuli. Then, if we consider between pairs, subjects had-at the end of the structural analysis of the faces - two configurational face representations that were clearly different: they could then easily answer "different" in the delayed same-different matching task. For same pairs, the two identical faces gave rise to a unique configurational face representation: subjects could then easily answer "same" in the delayed samedifferent matching task. The situation was different for within pairs: in fact, at the end of the structural analysis of the two presented faces, the same configurational face representation was activated because the two images represented the same identity. However, the two images are physically different. Then, to give a correct answer in the same-different matching task (i.e., "different"), subjects had to rely on the observed physical differences between the two images while inhibiting their "configurational equivalence." This explains why the task was more difficult when subjects were confronted with the within pairs as compared with the between and same pairs, this higher complexity being represented by a P $3 b$ of higher amplitude in the evoked brain response (at $\mathrm{Pz}$ ) and by a lower rate of performance in behavioral results.

\section{Conclusions}

The hallmark of categorical perception effect is a better discrimination of between-categorical differences as compared with withincategorical differences (Young et al., 1997). The present study determined the temporal course of face perceptual categorization: such categorization takes place early in the perceptual face processing, that is, at around $150 \mathrm{~ms}$ following stimulus onset. Discrimination of within-categorical differences is more complex because the two images of within pairs are physically different but give rise to an identical configurational face representation. This result was shown by the priming effect described on N170, around $156 \mathrm{~ms}$ at T6, for within and same pairs as compared with between pairs. Consequently, because our brain has to overcome the identity similarity of the two images in order to rely on their physical differences, the process that will lead subjects to give a correct answer ("different") to within pairs is more complex than the process used to arrive at correct answers for between and same pairs. This difference in processing was marked in behavioral results by the lower performance for within pairs and in ERPs by a $\mathrm{P} 3 \mathrm{~b}$ of higher amplitude for within pairs as compared with between and same pairs. It was then important to outline the complete consistency existing between ERP results and behavioral data.

\section{REFERENCES}

Allison, T., Puce, A., Spencer, D. D., \& McCarthy, G. (1999). Electrophysiological studies of human face perception. I: Potentials generated in occipitotemporal cortex by face and non-face stimuli. Cerebral Cortex, 9, 415-430.

Badgayian, R. D., \& Posner, M. I. (1996). Priming reduces input activity in right posterior cortex during stem completion. NeuroReport, 7, 2975-2978

Beale, J. M., \& Keil, C. F. (1995). Categorical effects in the perception of faces. Cognition, 57, 217-239.

Begleiter, H., Porjesz, B., \& Wang, W. Y. (1995). Event-related brain potentials differentiate priming and recognition to familiar and unfamiliar faces. Electroencephalography and Clinical Neurophysiology, 94, 41-49.

Bentin, S., Allison, T., Puce, A., Perez, E., \& McCarthy, G. (1996). Electrophysiological studies of face perception in humans. Journal of Cognitive Neuroscience, $8,551-565$.

Bentin, S., \& Deouell, L. Y. (2000). Journal of Cognitive Neuropsychology, 17, 35-54.

Bentin, S., Deouell, L. Y., \& Soroker, N. (1999). Selective visual streaming in face recognition: Evidence from developmental prosopagnosia. $\mathrm{Neu}$ roReport, 10, 823-827.

Bentin, S., Mouchetant-Rostaing, Y., Giard, M. H., Echallier, J. F., \& Pernier, J. (1999). ERP manifestations of processing printed words at different psycholinguistic levels: Time course and scalp distribution. Journal of Cognitive Neuroscience, 11, 235-260.

Bertrand, O., Perrin, F., \& Perrier, J. (1985). A theoretical justification of the average reference in topographic evoked potentials studies. Clinical Neurophysiology, 62, 462-464.

Blumstein, S., Cooper, W., Zurif, E., \& Caramazza, A. (1977). The per- ception and production of voice-onset-time in aphasia. Neuropsychologia, 15, 371-383.

Bornstein, M. H., \& Korda, N. O. (1984). Discrimination and matching within and between hues measured by reaction times: Some implications for categorical perception and levels of information processing. Psychological Research, 46, 207-222.

Bötzel, K., \& Grüsser, O. J. (1989). Electric brain potentials evoked by pictures of faces and non-faces: A search for "face specific" EEG potentials. Experimental Brain Research, 77, 349-360.

Bötzel, K., Schulze, S., \& Stodieck, R. G. (1995). Scalp topography and analysis of intracranial sources of face-evoked potentials. Experimental Brain Research, 104, 135-143.

Clarck, V. P., \& Hillyard, S. A. (1996). Spatial selective attention affects early extratriate components of the visual evoked potentials. Journal of Cognitive Neuroscience, 8, 387-402.

Comerchero, M. D., \& Polich, J. (1999). P3a and P3b from typical auditory and visual stimuli. Clinical Neurophysiology, 110, 24-30.

Dehaene-Lambertz, G., \& Dehaene, S. (1994). Speed and cerebral correlates of syllabe discrimination in infants. Nature, 370, 292-295.

De Renzi, E., Perani, D., Carlesimo, G. A., Silveri, M. C., \& Fazio, F. (1994). Prosopagnosia can be associated with damage confined to the right hemisphere-An MRI and PET study and a review of the literature. Neuropsychologia, 32, 893-902.

Dorman, M. (1974). Auditory evoked potential correlates of speech sound discrimination. Perception and Psychophysics, 15, 215-220.

Duncan-Johnson, C. C., \& Donchin, E. (1982). The P300 component of the event-related brain potential as an index of information processing. Biological Psychology, 14, 1-52. 
Eimer, M., \& McCarthy, R. A. (1999). Prosopagnosia and structural encoding of faces: Evidence from event-related potentials. NeuroReport, $10,255-259$.

George, N., Evans, J., Fiori, N., Davidoff, J., \& Renault, B. (1996). Brain events related to normal and moderately scrambled faces. Cognitive Brain Research, 4, 65-76.

Goldstone, R. L. (1998). Perceptual learning. Annual Review of Psychology, 49, 585-612.

Gomez, C. M., Clarck, V. P., Luck, S. J., Fan, S., \& Hillyard, S. A. (1994). Sources of attention-sensitive visual event-related potentials. Brain Topography, 7, 41-51.

Halgren, E., Baudena, P., Heit, G., Clarke, M., Marinkovic, K., \& Chauvel, P. (1994). Spatio-temporal stages in face and word processing. 2. Depthrecorded potentials in the human frontal and Rolandic cortices. Journal of Physiology, 88, 51-80.

Harnad, S. (Ed.). (1987). Categorical perception: The groundwork of cognition. Cambridge, UK: Cambridge University Press.

Heinze, H. J., Mangun, G. R., Burchert, W., Hinrichs, H., Scholtz, M., Münte, T. F., Gös, A., Scherg, M., Johannes, S., Hundeshagen, H., Gazzaniga, M. S., \& Hillyard, S. A. (1994). Combined spatial and temporal imaging of brain activity during selective attention in humans. Nature, 372, 543-546.

Hillger, L. A., \& Koenig, O. (1991). Separable mechanisms in face processing: Evidence from hemispheric specialization. Journal of Cognitive Neuroscience, 3, 42-57.

Jeffreys, D. A. (1989). A face-responsive potential recorded from the human scalp. Experimental Brain Research, 78, 193-202.

Jeffreys, D. A. (1996). Evoked potentials studies of face and object processing. Visual Cognition, 3, 1-38.

Jeffreys, D. A., \& Tukmachi, E. S. A. (1992). The vertex-positive scalp potential evoked by faces. Experimental Brain Research, 91, 340-350.

Jemel, B., George, N., Chaby, L., Fiori, N., \& Renault, B. (1999). Differential processing of part-to-whole and part-to-part face priming: An ERP study. NeuroReport, 10, 1069-1075.

Ji, J., Porjesz, B., \& Begleiter, H. (1998). ERP components in category matching tasks. Evoked Potentials-Electroencephalography and Clinical Neurophysiology, 108, 380-389.

Kanwisher, N., McDermott, J., \& Chun, M. M. (1997). The fusiform face area: A module in human extrastriate cortex specialized for face perception. Journal of Neuroscience, 17, 4302-4311.

Landis, T., Cummings, J. L., Christen, L., Bogen, J. E., \& Imhof, H.-G. (1986). Are unilateral right posterior cerebral lesions sufficient to cause prosopagnosia? Cortex, 22, 243-252.

Liberman, A. M., Harris, K. S., Hoffman, H. S., \& Griffith, B. C. (1957). The discrimination of speech sounds within and across phoneme boundaries. Journal of Experimental Psychology, 53, 368-385.

McCarthy, G., Puce, A., Gore, J. C., \& Allison, T. (1997). Face-specific processing in the human fusiform gyrus. Journal of Cognitive Neuroscience, 9, 605-610.

Molfese, D. L. (1987). Electrophysiological indices of categorical perception for speech. In Harnad, S. (Ed.), Categorical perception: The groundwork of cognition (pp. 421-443). New York: Cambridge University Press.

Paller, K. A., \& Gross, M. (1998). Brain potentials associated with per- ceptual priming vs explicit remembering during the repetition of visual word-form. Neuropsychologia, 36, 559-571.

Rapcsak, S. Z., Polster, M. R., Glisky, M. L., \& Comer, J. F. (1996). False recognition of unfamiliar faces following right hemisphere damage: Neuropsychological and anatomical observations. Cortex, 32, 593-611.

Ravden, D., \& Polich, J. (1998). Habituation of P300 from visual stimuli. International Journal of Psychophysiology, 30, 359-365.

Rohrbaugh, J. W., Donchin, E., \& Eriksen, C. W. (1974). Decision making and the P300 component of the cortical evoked response. Perception and Psychophysics, 15, 368-374.

Rossion, B., Campanella, S., Guerit, J. M., Debatisse, D., Liard, L., Gomez, C., Dubois, S., Delinte, A., Bruyer, R., \& Crommelinck, M. (1999). Task modulation of brain activity related to familiar and unfamiliar face processing: An ERP study. Clinical Neurophysiology, 110, 449-462.

Rossion, B., Delvenne, J. F., Debatisse, D., Goffaux, V., Bruyer, R., Crommelinck, M., \& Guerit, J. M. (1999). Brain activity related to normal and inverted face processing: An event-related potential study. Biological Psychology, 50, 173-189.

Schendan, H. E., Ganis, G., \& Kutas, M. (1998). Neurophysiological evidence for visual perceptual categorization of words and faces within $150 \mathrm{~ms}$. Psychophysiology, 35, 240-251.

Schweinberger, S. R. (1996). How Gorbachev primed Yeltsin: Analyses of associative priming in person recognition by means of reaction times and event-related potentials. Journal of Experimental PsychologyLearning, Memory and Cognition, 22, 1383-1407.

Schweinberger, S. R., Pfutze, E. M., \& Sommer, W. (1995). Repetition priming and associative priming of face recognition. Evidence from event-related potentials. Journal of Experimental Psychology-Learning, Memory and Cognition, 21, 722-736.

Stevenage, V. S. (1998). Which twin are you? A demonstration of induced categorical perception of identical twin faces. British Journal of Psychology, 89, 39-57.

Tanaka, J., Giles, M., Kremen, S., \& Simon, V. (1998). Mapping attractor field in face space: The atypicality bias in face recognition. Cognition, 68, 199-220.

Taylor, M. J., McCarthy, G., Saliba, E., \& Degiovanni, E. (1999). ERP evidence of developmental changes in processing of faces. Clinical Neurophysiology, 110, 910-915.

Valentine, T. (1991). A unified account of the effects of distinctiveness, inversion, and race in face recognition. The Quarterly Journal of Experimental Psychology, 43A, 161-204.

Wilson, G. F., Swain, C. R., \& Ullsperger, P. (1998). ERP components elicited in response to warning stimuli: The influence of task difficulty. Biological Psychology, 47, 137-158.

Young, A. W., Rowland, D., Calder, A. J., Etcoff, N. L., Seth, A., \& Perrett, D. I. (1997). Facial expression megamix: Tests of dimensional and category accounts of emotion recognition. Cognition, 63, 271-313.

Zhang, X. L., Begleiter, H., Porjesz, B., \& Litke, A. (1997). Visual object priming differs from visual word priming: An ERP study. Electroencephalography and Clinical Neurophysiology, 102, 200-215.

(RECEIVED September 27, 1999; ACCEPTED January 4, 2000) 